\title{
MIMO Modeling Approach for a Small Photovoltaic Reverse Osmosis Desalination System
}

\author{
A.B. Chaaben ${ }^{1}$, R. Andoulsi ${ }^{2}$, A. Sellami ${ }^{3}$ and R. Mhiri ${ }^{4}$ \\ ${ }^{1}$ Research Unit RME, INSAT, North urban center, BP 676, 1080 Tunis, Tunisia \\ ${ }^{2}$ LaNSE/CRTEn Borj Cedria BP95, Hammam Lif, 2050 Tunis, Tunisia \\ ${ }^{3}$ ESSTT, 05 AV Taha Hussein Montfleury, 1008, Tunis, Tunisia \\ ${ }^{4}$ Sciences faculty of Tunis, 1020, Belvédère, Tunis, Tunisia \\ Email:Abd.chaabene@yahoo.fr
}

(Received January 5, 2009; accepted September 21, 2009)

\begin{abstract}
The most widely used desalination processes are based on membrane separation via reverse osmosis (RO) which has become an important process for desalting seawater and cleaning brackish water. The use of these processes requires an efficient control system. Consequently, it is necessary to establish a dynamic model of the system with experimental validation. This paper deals with a new modelling approach of a small photovoltaic reverse osmosis (PV-RO) desalination unit. The proposed model considers the unit as a Multi Input Multi Output (MIMO) process. The relations between the output variables and the input variables are given by the use of empirical transfer matrix. A state model of the unit is also given. Some experimental results are presented to validate the proposed model. As result, the obtained unit model can be easily used for a process control loop implementation in order to assure an optimum operating condition and to reduce the water product cost.
\end{abstract}

Keywords: Modelling; Photovoltaic; MIMO process; Desalination; Reverse osmosis, Renewable energy.

\section{NOMENCLATURE}

$\begin{array}{ll}\mathrm{K}_{\mathrm{ij}} & \text { Process gain; unitless } \\ \tau_{\mathrm{ij}} & \text { Time constant; } \mathrm{s} \\ \xi^{1,2} & \text { Damping coefficient; unitless } \\ \omega_{01,02} & \text { Natural throbbing; rad.s-1 } \\ \Pi & \text { Osmotic pressure; Pa } \\ \Omega_{\mathrm{p}} & \text { Angular pump speed; rps } \\ \theta_{\mathrm{vr}} & \text { Reject brine valve aperture; rad. } \\ \mathrm{Q}_{\mathrm{s}} & \text { Product water flow; } \mathrm{m}^{3} \cdot \mathrm{s}^{-1} \\ \mathrm{P} & \text { Pressure; } \mathrm{Pa}\end{array}$

\section{INTRODUCTION}

Water can be considered as one of the basic elements supporting life and the natural environment, a primary component for industry, and a consumer item for humans and animals. Consequently to improve as maximum as possible its quality we need to implement automated control techniques to make easily the management of water resources and decision making process. The control procedure requires the development of systems models. In Tunisia the quality of potable water is a critical problem especially in the southern part of the country (salinity ranging is between
Cs Product water salinity; ppm

$C_{e} \quad$ Feed water salinity

$T_{e} \quad$ Pump torque; N.m

$\mathrm{T}$ Temperature; $\mathrm{K}$

Qe Feed water flow; $\mathrm{m}^{3} \cdot \mathrm{s}^{-1}$

$\mu \quad$ Chemic potential; J.mol ${ }^{-1}$

$V \quad$ Solution volume $; \mathrm{m}^{3}$

Di Diffusion coefficient $; \mathrm{m}^{2} \cdot \mathrm{s}^{-1}$

$\mathrm{R} \quad$ Molar constant; J.mol ${ }^{-1} \cdot \mathrm{K}^{-1}$

(1.000-3.000 ppm) Bouguecha (2004), the use of small scale PV-RO desalination units (product capacity less than $2 \mathrm{~m}^{3} / \mathrm{d}$ ) represent a possible alternative for potable water supply for such areas where there is no access to electricity grid. The main reason for this is the poor energy efficiency that is typical of small scale PV-RO systems.

The desalination is a highly complex process that requires the knowledge of several parameters such as the pressure, the flow rate, $\mathrm{pH}$, temperature, salt concentration etc. (Awed et al., 2002). Therefore, the desalination unit is considered as a Multivariable system. 
Because of the concern for energy consumption, it is becoming increasingly important to operate the desalination units as close as possible to target Alatiqui et al. (1999). Since advanced control systems are keys to improve operation of these units, there has been a growing interest in recent years to present different mathematic models.

Several preliminary studies can be found in the literature, where many approaches of desalination units modelling are given. Alatiqui et al. (1999) have described a model for the sea water desalination systems where the manipulated variables are $\mathrm{pH}$ and feed pressure.

Calangelo et al. (1999) have described a mathematical model so that the governing equations can be solved using average hourly solar insulation and ambient temperature value. The application of this theoretical model to define a control strategy taking into account the temperature as a manipulated variable is difficult.

Mandler (2000) has developed a Single In Single Out (SISO) model for the small reverse osmosis desalination systems. Although this model can be used for control techniques, it does not allow the control of the salinity which is the most important quality parameter.

Robertson et al. (1996) have developed a dynamic matrix control algorithm based on the manipulation of the $\mathrm{pH}$ and the pressure. This later was used to drive a sea water reverse osmosis desalination pilot plant. However, this approach cannot be used in the case of RO plant for brackish water desalination. In fact, the salinity is less than that of the sea water and the variation of $\mathrm{pH}$ is not remarkable.

Thomson and Infield (2002) have presented a MatlabSimulink model of a PV-RO system but without battery. They have developed a maximum power point tracking for the photovoltaic array. The application of the desalination in line of sun is still difficult. Indeed; the variation of the period of sunshine provokes the sealing of membranes.

Ballannec et al. (1999) have presented a representative modelling with salt solutes based on an experimental study. The model presents convection-diffusion equations associated with the osmotic theory equations and describes the mass transfer mechanism in the polarisation layer. This model with non linear equations cannot be used to implement classical automated control techniques which need the introduction of manipulated variables.

However the use of all these respect models to develop an advanced control strategies for the RO desalination plants presents also some difficulties due to either the choice of the system parameters which should be monitored and controlled or to the limited number of these parameters such as the SISO models.

The present study gives a new approach of modelling. It's firstly based on experimental results, thus the choice of the manipulated parameters was tested. Secondly, it takes into account the mathematic formulation of the solubilisation-diffusion phenomenon. Finally, it presents the unit as a Multi
Input Multi Output system which is the nearest representation of the system dynamic.

This paper is organized as follows: First, we present, a description of the desalination system and the RO phenomenon, then we give the equivalent diagram of the energetic system.

The fifth section is devoted to the modelling of the RO unit where two models are given: the transfer model and the state space model.

The last section is dedicated to the validation and discussion of results.

\section{DESALINATION SYSTEM DESCRIPTION}

The desalination system that we present is composed by:

- A photovoltaic generator

- A DC/AC converter

- Accumulator batteries

- The RO desalination unit

The system configuration is shown in Fig. 1 is composed by three compartments: the photovoltaic generator, the electrical adaptor and the desalination unit

The PV generator consists of an array of photovoltaic cell modules connected in series-parallel combination to provide the desired DC voltage and current.

The adaptor converts the continuous voltage delivered by the battery to an alternative voltage in order to feed the motor pump group which provides pressurized water to the membrane.

The desalination unit is composed by three RO modules, each one of them is composed by a membrane which is constituted of a thin film composite modified polyamide type able to purify feed water containing up to 3,000 ppm of total dissolved solids. These modules have a nominal capacity $1500 \mathrm{~L} / \mathrm{d}$ at $800 \mathrm{kPa}$.

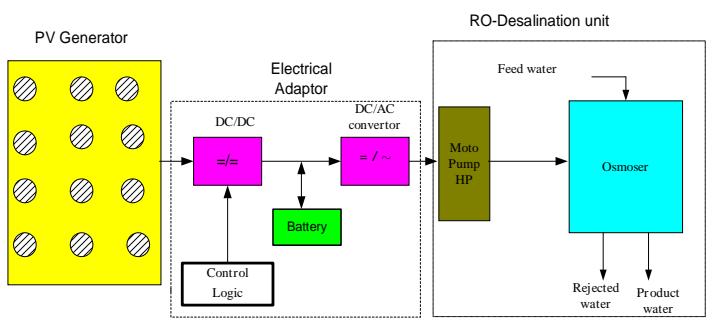

Fig. 1. Desalination system coupled to a photovoltaic generator

\section{REVERSE OSMOSIS}

\subsection{Principle}

In order to determine the input and the output variables of the desalination unit, we present briefly the reverse osmosis principle. 
When we try to separate pure water and a salt solution through a semipermeable membrane, the pure water diffuses through the membrane and dilutes the salt solution. The membrane rejects most of the dissolved salts, while allowing the water to permeate.

This phenomenon is known as natural or direct osmosis. As water passes through the membrane, the pressure on the dilute side drops, and the pressure of the concentrated solution rises. The osmotic flux continues until an equilibrium is reached, where the net water flux through the membrane becomes zero at equilibrium; the liquid level in the saline water will be higher than that on the water side. The amount of water passing in either direction achieved is equal to the effective driving force causing the flow, called osmotic pressure noted $\mathbf{I}$ which is due to the difference between the salt concentration of both of the feed and the product water. This pressure is a strong function of the solute concentration and the temperature, and depends on the type of ionic species present. Applying a pressure $\mathbf{P}$ in excess of the osmotic pressure to the saline water section slows down the osmotic flow, and forces the water to flow from the salt solution into th- water side. Thereforg, the direction of flow is reversed and that is why this separation process is called reverse osmosis. (Fig. 2).

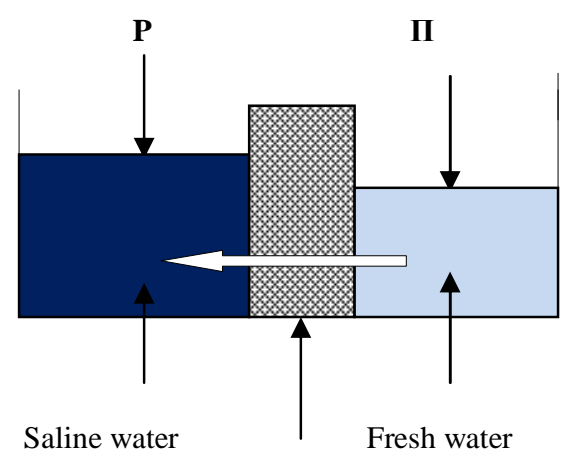

Membrane

(a) $\mathbf{P}<$ I

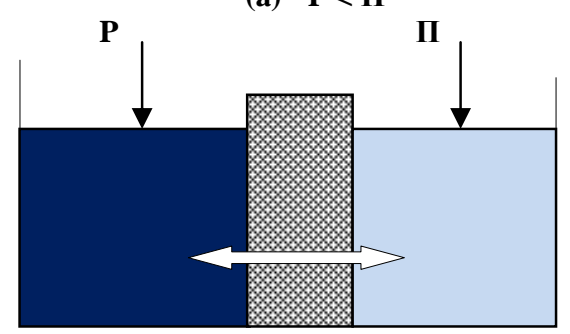

(b) $\mathbf{P}=\mathbf{\Pi}$

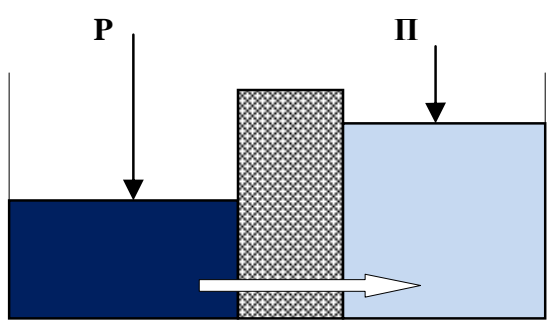

(c) $\mathbf{P}>$ II

Fig. 2. Principle of the reverse osmosis: (a) natural osmosis, (b) osmotic equilibrium, (c) reverse osmotic

\subsection{Mathematical Formulation}

In order to evaluate the component (i) flow through the osmosis membrane, the used model is a solubilisation diffusion model. Sure enough both of the solvent and solute flows $Q_{i}$ are represented by the following equations:

$Q_{i}=\frac{-D_{i} C_{i}}{R T} \operatorname{grad}\left(\mu_{i}\right)$

$Q_{i}=\frac{-D_{i} C_{i}}{R T}\left(\frac{\partial \mu_{i}}{\partial C_{i}} \operatorname{grad}\left(C_{i}\right)+\operatorname{Vgrad}(P)\right)$

In the case of the solvent, water in our application, the term of grad $\left(\mathrm{C}_{\mathrm{i}}\right)$ is negligible thus the water flow through the membrane is given by the following equation:

$Q_{i}=\frac{D C V}{R T}\left(\frac{\Delta P-\Delta \pi}{\Delta X}\right)$

For the solute, the term of $\mathrm{V}$. $\operatorname{grad}(\mathrm{P})$ is negligible and the solute flow is given by the following equation

$Q_{i}=-D \cdot K \frac{\Delta C}{\Delta X}=B\left(C_{e}-C_{s}\right)$

\section{Photovoltaic Generator Model}

The use of PV generators makes easily the use of solar energy, free, clean and inexhaustible. For the best exploitation of this energy and for a continuous production of water, we must install the recharged batteries Richards et al. (2002).

The coupling of the battery with the PV generator is realized through a charge regulator. The equivalent electrical diagram of the total energetic system is shown in Fig. 3.

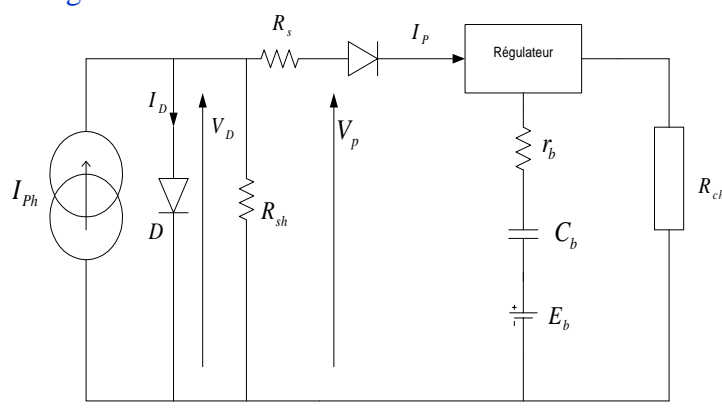

Fig. 3. The energetic system equivalent diagram

The corresponding I-V characteristic, strongly non linear can be represented by the following equations (Sellami et al., 1998)

$$
\begin{aligned}
& V_{p}=V_{D}-R_{S} I_{p} \\
& I_{p}=I_{p h}-I_{D}-\frac{V_{D}}{R_{S h}} \\
& I_{p}=I_{p h}-I_{s}\left[\exp \left(\frac{q\left(V_{p}+R_{s} I_{p}\right)}{A K T}\right)-1\right]-\frac{V_{p}+R_{s} I_{p}}{R_{s h}}
\end{aligned}
$$

Where $I_{p}$ is the photo current, $I_{s}$ is the reverse saturation current, $q$ is the elementary charge, $V_{p}$ is the terminal 
voltage, $R_{s h}$ is the shunt resistance caused by the cell surface state, $R_{S}$ is the serial resistance caused by the based resistance of the junction face, $A$ is the idealist factor, $\mathrm{K}$ is the Boltzmann constant and $T$ is the absolute temperature.

For the battery there are several models in literature among them we carry this presented by Borowy et al. (1997) relative to a Plumb battery where the equivalent electrical diagram is composed by a voltage generator coupled in series with an internal resistance $r_{b}$ and a capacity $c_{b}$ which represent the electrical charge and discharge of the battery.

\section{The Desalination Unit Model}

Because of the variety of parameters, the present study proposes the introduction of a new model which presents the brackish water desalination small unit as a multi Input Multi Output (MIMO) system. The output variables or set variables are the product flow $\boldsymbol{Q}_{s}$ and the product water salinity $\boldsymbol{C}_{\boldsymbol{s}}$. These two parameters are fundamental to control water quality Thomson (2002), while the input variables or manipulated variables are the motor pump angular speed $\boldsymbol{\Omega} p$ and the reject valve opening $\boldsymbol{\theta}_{\boldsymbol{v}}$. The water feed salinity $\boldsymbol{C}_{\mathrm{e}}$ is considered as a disruptive input. The choice of these parameters is based on the experimental results that we'll present in this paper. These results show the dependence of the water quality with these parameters.

In addition to these variables, there are others which may need to be monitored or controlled such the feed $\mathrm{pH}$ and temperature. The present study does not address the influence of these additional variables. Then, our interest is to develop a representative model for the RO desalination unit only which is the principal part of the whole system.

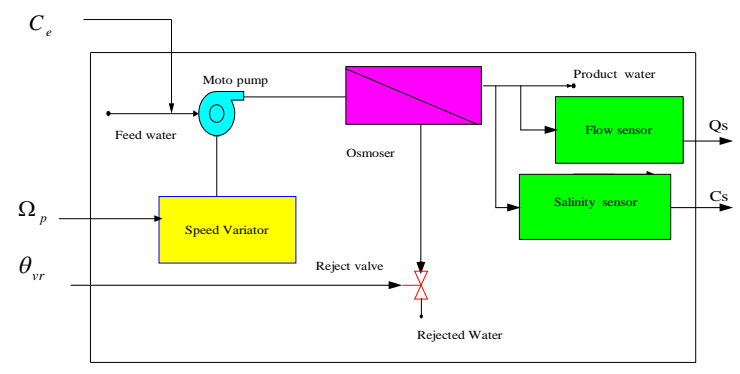

Fig. 4. The desalination unit configuration.

The choice of the motor pump angular speed $\boldsymbol{\Omega} \boldsymbol{p}$ as an input variable is based on the relation between the speed $\boldsymbol{\Omega}_{p}$, the water flow $\boldsymbol{Q}$ and the pump torque

$T_{e}=K \boldsymbol{\Omega}_{p} \boldsymbol{Q}$

The pressure $P$ applied at the membrane is

$P=K \boldsymbol{\Omega}_{p}{ }^{2}$

Where $K$ is a constant depending with the pump characteristics and the fluid density.

The following figure shows the unit static model which presents the input and output variables.

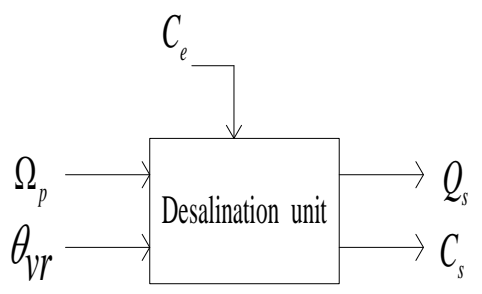

Fig.5. The unit static model

\subsection{The Desalination Unit Transfer Model}

The unit dynamic model is given by a transfer function matrix. The relations between output variables and input variables are based on elementary first or second order transfer functions noted $\boldsymbol{G}_{i j}$ Robertson et al. (1996). This MIMO process, with $m=2$ input variables and $n=2$ output variables, has the following representation:

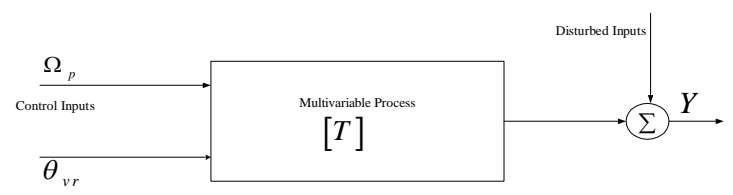

Fig. 6. MIMO structure of the process

Where the outputs vector $\mathrm{Y}$ and the control vector $\mathrm{U}$ are given by the following representations:

$Y=\left[\begin{array}{l}Q_{s} \\ C_{s}\end{array}\right]$ et $\quad U=\left[\begin{array}{c}\Omega_{p} \\ \theta_{v r}\end{array}\right]$

The transfer matrix [T] is represented by:

$[T]=\left[\begin{array}{ll}G_{11} & G_{12} \\ G_{21} & G_{22}\end{array}\right]$

Thus, the process will be described by the following equation:

$Y=[T] U$

In order to determine the elementary transfer functions, we have realized a serial of experimental measurements using a control and a data acquisition system. Then, we have used the identification procedure "ident " based on the recursive least square with Matlab software, Zilouchian (2001).

The $\boldsymbol{G}_{i j}$ expressions are:

$G_{11}=\left(\frac{Q_{s}}{\Omega_{p}}\right)_{\theta_{v r}, C_{e}=c t e}=\frac{k_{11}}{1+\tau_{11} p}$

$G_{12}=\left(\frac{Q_{s}}{\theta_{v r}}\right)_{\Omega_{p}, C_{e}=c t e}=\frac{k_{12} \omega_{\theta_{1}}^{2}}{p^{2}+2 \xi_{1} \omega_{0_{1}} p+\omega_{1}^{2}}$

$G_{21}=\left(\frac{C_{s}}{\Omega_{p}}\right)_{\theta_{r}, C_{e}=c t e}=\frac{k_{21} \omega_{2}^{2}}{p^{2}+2 \xi_{2} \omega_{2} p+\omega_{b_{2}}^{2}}$ 
$G_{22}=\left(\frac{C_{s}}{\theta_{v r}}\right)_{\Omega_{p}, C_{e}=c t e}=\frac{k_{22}}{1+\tau_{22} p}$

The parameters values given by the identification procedure with the disturbed parameter fixed at its least value in practical conditions $C_{\mathrm{e}}=1,000 \mathrm{ppm}$ and its maximum value $C_{\mathrm{e}}=3,000 \mathrm{ppm}$ as in Table 1 .

Table 1 Model parameters values at $\mathrm{C}_{\mathrm{e}}=1 \mathrm{~g} / \mathrm{L}$ and $\mathrm{C}_{\mathrm{e}}=3 \mathrm{~g} / \mathrm{L}$

\begin{tabular}{|c|c|c|}
\hline Parameters & $\begin{array}{c}\text { Values at } \\
\mathrm{C}_{\mathrm{e}}=1 \mathrm{~g} / \mathrm{L}\end{array}$ & $\begin{array}{c}\text { Values at } \\
\mathrm{C}_{\mathrm{e}}=3 \mathrm{~g} / \mathrm{L}\end{array}$ \\
\hline $\mathrm{k}_{11}$ & 2.50 & 3.00 \\
\hline$\tau_{11}$ & 1.00 & 1.10 \\
\hline $\mathrm{k}_{22}$ & -0.20 & -0.16 \\
\hline$\tau_{22}$ & 1.00 & 1.10 \\
\hline $\mathrm{k}_{12}$ & -0.25 & -0.28 \\
\hline$\omega_{01}$ & 1.50 & 1.20 \\
\hline$\xi_{1}$ & 0.50 & 0.30 \\
\hline $\mathrm{k}_{21}$ & -0.17 & -0.20 \\
\hline$\omega_{02}$ & 2.15 & 1.72 \\
\hline$\xi_{2}$ & 0.75 & 0.45 \\
\hline
\end{tabular}

The analysis of these expressions shows that the motor pump speed $\boldsymbol{\Omega}_{\mathrm{p}}$ has a negative action on the water product salinity, but a positive action on the water flow according to Eq. (3) and Eq. (4).

The reject valve opening has also a negative action on the water product flow as

$Q_{s}=Q_{e}-Q_{r}$

Where $\boldsymbol{Q r}$ is the reject flow and $\boldsymbol{Q e}$ is the feed water flow.

This last expression shows the effect of the reject valve opening on the direction of the change of the product and the reject flow rates.

\subsection{The Desalination Unit State Space Model}

The input variables are disconnected thus the process can be represented by the following state equation:

$$
\left\{\begin{array}{l}
\dot{X}=A X+B U \\
Y=C X
\end{array}\right.
$$

Where the state vector $\boldsymbol{X}$ and The control vector $\boldsymbol{U}$ are defined as

$$
X=\left(\begin{array}{c}
Q_{s} \\
\dot{Q}_{s} \\
C_{s} \\
\dot{C}_{s}
\end{array}\right), \quad U=\left[\begin{array}{c}
\Omega_{p} \\
\theta_{v r}
\end{array}\right]
$$

The constants matrices $\mathrm{A}$ and $\mathrm{B}$ are defined as

$$
A=\left(\begin{array}{cccc}
-\frac{1}{\tau_{11}} & 1 & 0 & 0 \\
-\omega_{0_{1}}^{2} & -2 \xi_{1} \omega_{0_{1}} & 0 & 0 \\
0 & 0 & -\frac{1}{\tau_{22}} & 1 \\
0 & 0 & -\omega_{0_{2}}^{2} & -2 \xi_{2} \omega_{0_{2}}
\end{array}\right)
$$

$$
B=\left(\begin{array}{cc}
\frac{k_{11}}{\tau_{11}} & 0 \\
0 & k_{12} \omega_{0_{1}}^{2} \\
0 & \frac{k_{22}}{\tau_{22}} \\
k_{21} \omega_{0_{2}}^{2} & 0
\end{array}\right)
$$

If we replace the parameters with their values at the least value of the feed salinity $C \mathrm{e}=1,000 \mathrm{ppm}$ we obtain

$$
\begin{aligned}
& A=\left(\begin{array}{cccc}
-1 & 1 & 0 & 0 \\
-2.25 & -1.50 & 0 & 0 \\
0 & 0 & -1 & 1 \\
0 & 0 & -4.62 & -3.23
\end{array}\right) \\
& B=\left(\begin{array}{cc}
2.50 & 0 \\
0 & -0.56 \\
0 & -0.20 \\
-0.81 & 0
\end{array}\right)
\end{aligned}
$$

The state matrix $\mathrm{C}$ is defined as

$C=\left(\begin{array}{llll}1 & 0 & 0 & 0 \\ 0 & 0 & 1 & 0\end{array}\right)$

The choice of the least value of $C_{\mathrm{e}}$ to valid the given model is based on the quality of the brackish water in the region where the unit was installed. However, to model the disturbed input we must develop an uncertain model which takes in to account the two extreme values of the feed salinity and determine consequently the matrices of parametric disturbance. Thus, the parametric disturbance has an important influence on the state matrix values.

\section{EXPERIMENTAL RESULTS MODELS VALIDATION}

\subsection{Results and Discussion}

In order to have an idea about relations between inputs and outputs variables, we have realized at the first time several measure serials using a control and a data acquisition system. In each measure serial, one of the inputs is variable but the others are taken constants Ballannec et al. (1999).

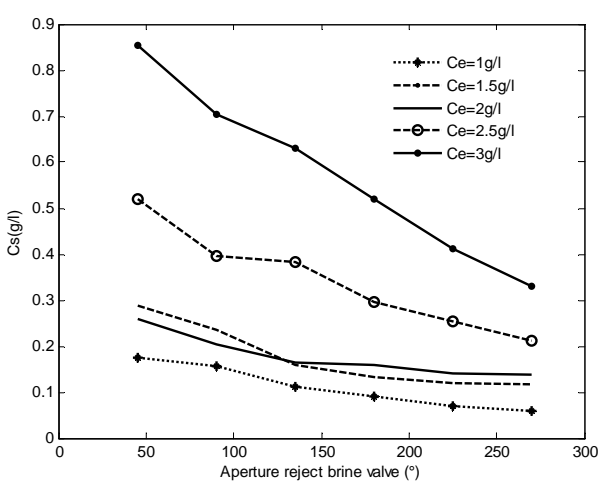

Fig. 7. Product water salinity vs aperture reject brine valve for various feed salinity for $\Omega_{\mathrm{p}}=18 \mathrm{rps}$

Figures 7, 8 and 9 show that the product water salinity $\boldsymbol{C}_{\boldsymbol{s}}$ decreases with the aperture reject brine valve $\boldsymbol{\theta}_{\boldsymbol{v} r}$ and 
the motor pump speed $\boldsymbol{\Omega}_{p}$, consequently these two input variables have a negative action at $\boldsymbol{C s}$ according to the expressions Eq. (14) and Eq. (15) where $\mathrm{k}_{21}<0$ and $\mathrm{k}_{22}$ $<0$.

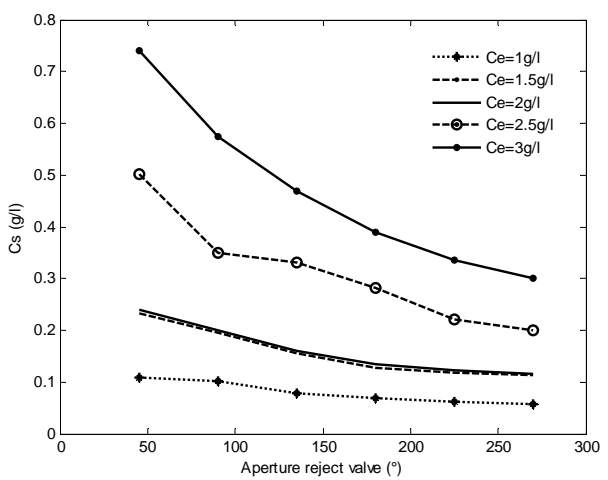

Fig. 8. Product water salinity vs aperture reject brine valve for various feed salinity for $\Omega_{\mathrm{p}}=24 \mathrm{rps}$

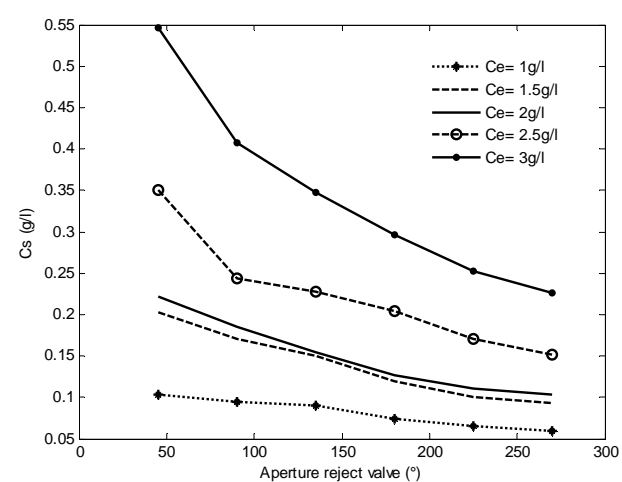

Fig. 9. Product water salinity vs aperture reject brine valve for various feed salinity for $\Omega_{\mathrm{p}}=30 \mathrm{rps}$

Figure 10 shows that the motor pump speed has a positive action on the water product flow, this is confirmed by the Eq. (3). The aperture reject brine valve has a negative on the product water flow from a position where the product flow is maximum B. Chaabene (1994) The product water flow decreases in all cases with feed salinity.

\subsection{Models Validation}

To valid the established models, we have applied step type signals for the input variables than we take the output values with the data acquisition system. In order to minimize the difference between the model and the experimental data, the developed acquisition software calculates the average of values serial of each parameter and stores them in files. Then, these files of measured values are used by Identification MATLAB Toolbox.

Figures 11 and 12 valid the $\boldsymbol{Q s}$ and the $\boldsymbol{C s}$ models when a profile of step input is applied to the motor pump speed $\boldsymbol{\Omega}_{p}$, the aperture reject brine is fixed at the optimum value given by the Fig. 10 .

Then we have applied a profile of a step input for the aperture reject brine valve, the motor pump speed was fixed at its nominal value $\boldsymbol{\Omega}_{\boldsymbol{p}}=30 \mathrm{rps}$.

Figures 13 and 14 show the validation of the $Q s$ and the Cs models.

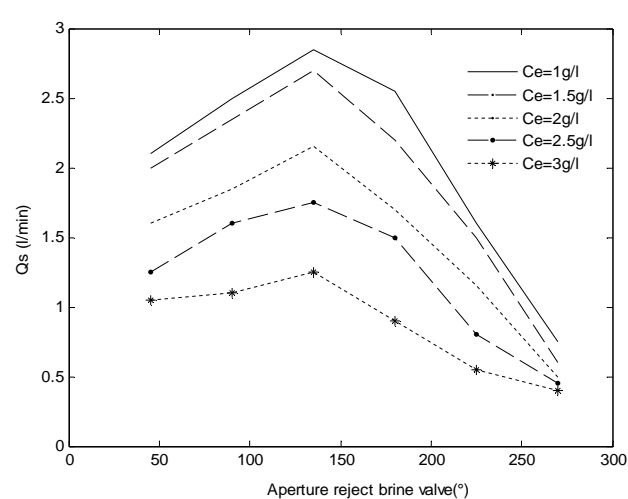

Fig. 10. Product flow vs aperture brine valve for various feed salinity at $\Omega_{\mathrm{p}}=18 \mathrm{rps}$

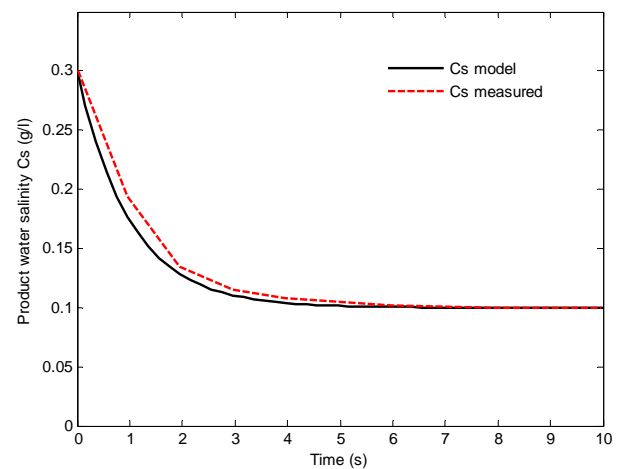

Fig. 11. Cs model validation on a step response of the reject valve aperture

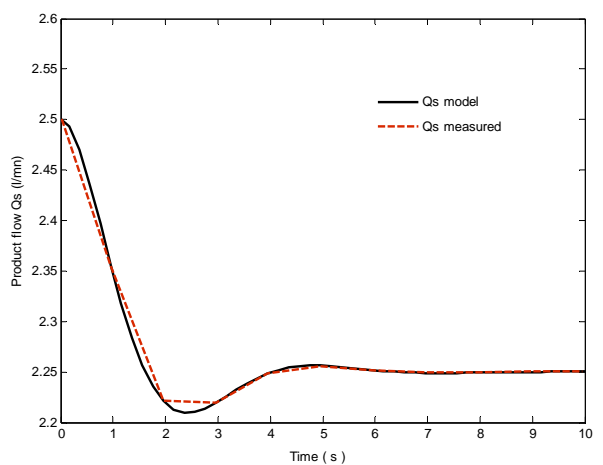

Fig. 12.Qs model validation on a step response of the reject valve aperture

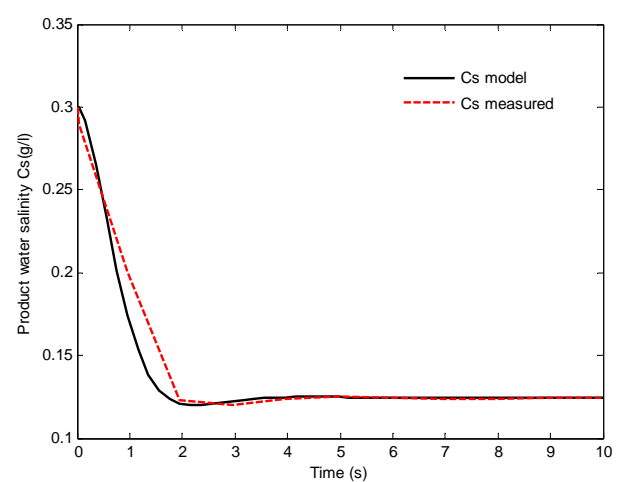

Fig. 13. Cs model validation on a step response of the motor pump speed 


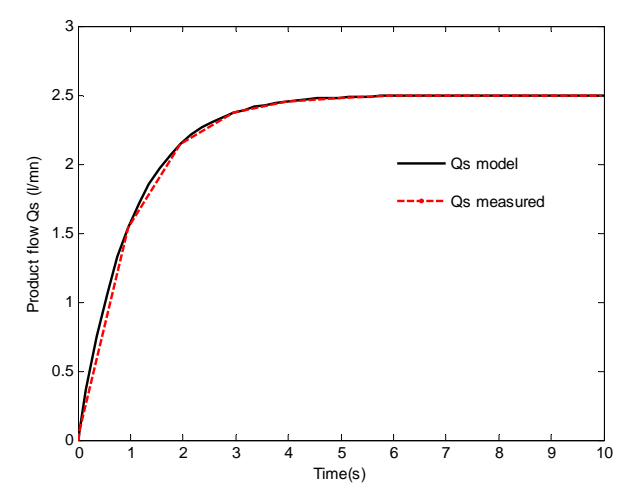

Fig. 14. Qs model validation on a step response of the motor pump speed

\section{CONCLUSION}

In this work we have developed a Multi Input Multi Output model for the dynamic of the desalination unit. The presented model shows the effect of the disturbed input at the system dynamic.

To establish this model we are interested in the two essential quality parameters of the product water which are the product water flow and the product water salinity. The experimental results show clearly the effect of the manipulated variables on the system behaviour.

The development of control strategies for the desalination system will be simplified by the use of each of the transfer model or the state space model.

An immediate extension of this study is the development of a general model which gathers all parts of the system. The state space model must be uncertain to take in to account the parametric disturbance.

\section{REFERENCES}

Alatiqui, I., H. Ettouney and H. EL-Dessouky (1999). Process control in water desalination industry: an overview. Desalination 126, 15-32.

Ballannec, B., S. Nicolas and B. Bariou (1999). Experimental study and modelisation of reverse osmosis with salt solutesin an unstirred batch cell. Desalination 122, 43-51.

Borowy, B.S. and Z.M. Salameh (1997). Dynamic response of a stand- alone wind energy conversion system with battery storage to a wind gust. IEEE Transaction on Energy Conversion 12, 73-75.

Bouguecha, S., B. Hamrouni and M. Dhahbi, (2004). Operating analysis of a direct energy coupled desalination family prototype. Desalination 168, 95-100.

Chaâbène, A.B., S. Bouguecha, A. Guizani and M. Annabi (1994). Automatic control of a reverse osmosis desalination unit. Proceedings of the first Maghreb Conference of process engineering 1, 5255 .

Colangelo, A., D. Marano, G. Spagna and V.K. Sharma (1999). Photovoltaic powered reverse osmosis water desalination. International Journal of Applied Energy 64, 289-305.

Dababneh, A.J. and M.A. AL-Nimr (2002). A reverse osmoses desalination unit. Desalination 153, 265272.

Mandler, J.A. (2000). Modelling for control analysis and design in complex industrial separation and liquefaction processes. Journal of process control 2, 167-175.

Richards, B.S. and A.I. Schafer (2002). Design consideration for a solar powered desalination system for remote communities in Australia. Journal of Desalination 144, 193-199.

Robertson, M.W., J.C. Watters, P.B. Desphande, J.Z. Assef and I. Alatiqui (1996). Model based control for reverse osmosis desalination process. Desalination 104, 59-68.

Sellami, A., F. Ghodbane, R. Andoulsi and H. Ezzaouia (1998, September). An Electrical Performance Tester for PV Modules. Proceeding of World Renewable Energy Congress 15-16/4003, 17171719.

Thomson, M. and D. Infield (2002). A photovoltaicpowered sea water reverse osmosis system. Desalination 153, 1-8.

Zilouchian, A. and M. Jafar (2001). Automation and process control for a reverse osmosis plants using soft computing methodology. Desalination 135, $51-5$ 